\title{
Recognition memory ROCs for item and associative information: The contribution of recollection and familiarity
}

\author{
ANDREW P. YONELINAS \\ University of California, Davis, California
}

\begin{abstract}
Receiver-operating characteristics (ROCs) were examined in three recognition memory experiments. ROCs for item information (i.e., was this word presented?) were found to be curvilinear. However, ROCs for associative information (i.e., were these two words presented together?) were found to be linear. The results are in agreement with the predictions of a dual-process model that assumes that recognition judgments are based on familiarity and recollection. Familiarity reflects the assessment of a continuous strength dimension and is well described as a signal detection process, whereas recollection reflects the retrieval of qualitative information about the study episode and behaves like a discrete threshold process. The results showed that memory judgments about items relied on a combination of recollection and familiarity, but that judgments about associations relied primarily on recollection. Further examination of the associative ROCs suggested that subjects were able to recollect that old pairs of items were in the study list, and, under some conditions, that new pairs were not in the study list.
\end{abstract}

The analysis of receiver-operating characteristics (ROCs) has played a critical role in the development of memory theories for several decades. An ROC is the function that relates hits (i.e., correct recognitions) to false alarms (i.e., incorrect recognitions). In the early $1960 \mathrm{~s}$, the examination of ROCs revealed that the relation between hits and false alarms was almost always nonlinear, and this led many researchers to reject an entire class of linear "threshold" models (see Murdock, 1974). Although almost all of the current memory models now produce curvilinear ROCs, Ratcliff, Sheu, and Gronlund (1992) have recently shown that none of the current models, including distributed and localized storage models, provide an adequate account of the observed ROCs. In response to these findings, a dual-process model was developed that could account for the ROC data (Yonelinas, 1994). The model assumes that recognition judgments can be based on the assessment of a continuous familiarity dimension (as is assumed in most other models) but that an independent recollection process also contributes to performance. In the present paper, I examine the necessity of postulating a recollection process in recognition memory and test a number of predictions based on the dual-process model by examining recognition ROCs for item and associative information.

I will begin by describing the existing ROC data and show why it raises problems for current models; then I will describe the dual-process signal detection model and

I wish to thank Steven Clark, William Hockley, Geoffrey Loftus, and Bennet Murdock for their comments and criticisms of earlier versions of this manuscript. Correspondence should be sent to A. P. Yonelinas, Department of Psychology, University of California. Davis. CA 95616 (e-mail: apyonelinas@ucdavis.edu). show how it accounts for the existing data. I will then discuss an unequal-variance signal detection model that is also in general agreement with the ROC data, and contrast it with the dual-process model. I will then test the two models by examining ROCs for item and associative information in three recognition memory experiments.

\section{Recognition Memory ROCs}

One of the most direct ways of testing theories of recognition memory is to determine whether they can accurately account for the relationship between hits and false alarms (i.e., ROCs). ROC data can be collected in several different ways, but typically subjects are required to rate the confidence of their recognition judgments, and performance is plotted as a function of response confidence. For example, the first point on the function is determined by adopting a very strict response criterion; only the most confident "yes" responses are included as hits and false alarms. The second point reflects a slightly more relaxed response criterion; the most confident "yes" responses as well as the second most confident "yes" responses are treated as hits and false alarms. By examining performance at a number of different levels of response confidence, one can plot the ROC and the relationship between hits and false alarms can be studied.

Recognition memory ROCs tend to look like those presented in the left panels of Figures $1 b$ and $1 c$. The functions increase gradually in a curvilinear manner, and they approach the 1,1 intercept. The ROCs are curvilinear, and they are typically asymmetrical along the negative diagonal (compare Figures $1 \mathrm{a}$ and $1 \mathrm{~b}$ ). Importantly, the degree to which the curves are asymmetrical varies across different experimental manipulations. To measure the asymmetry one can replot the ROCs on $z$-coordinates ( $z$-ROCs) and es- 
a) Familiarity
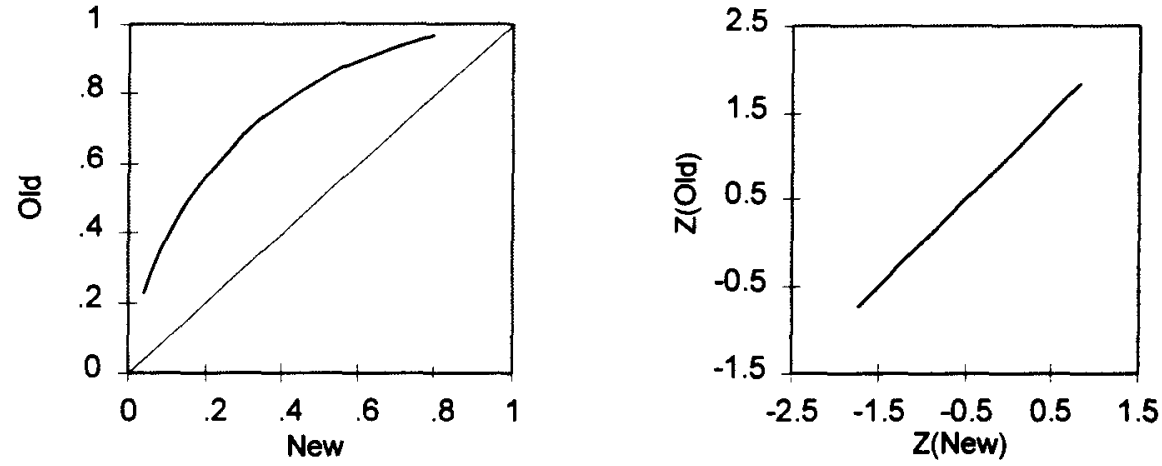

b) Familiarity and Recollection
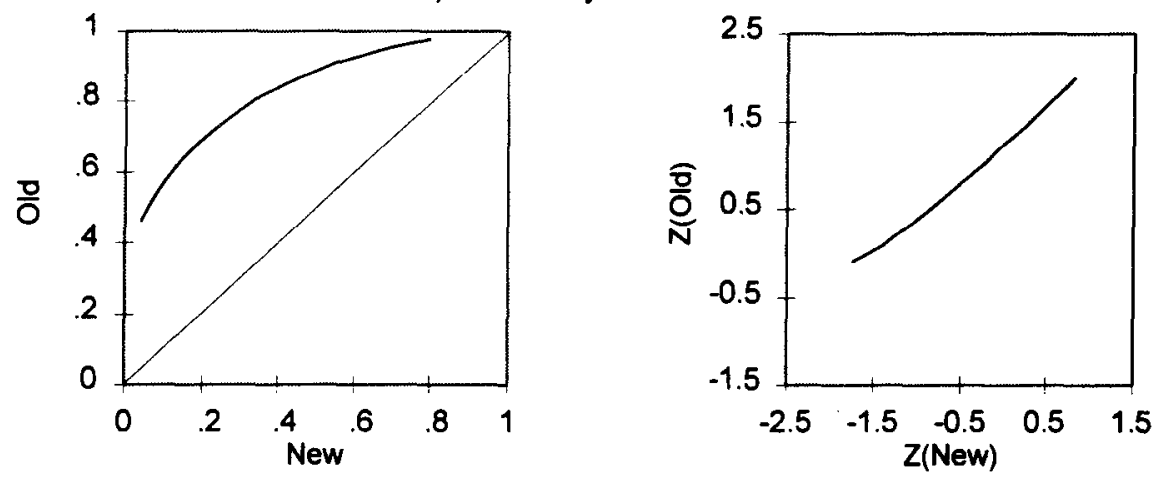

c) Unequal-Variance SDT
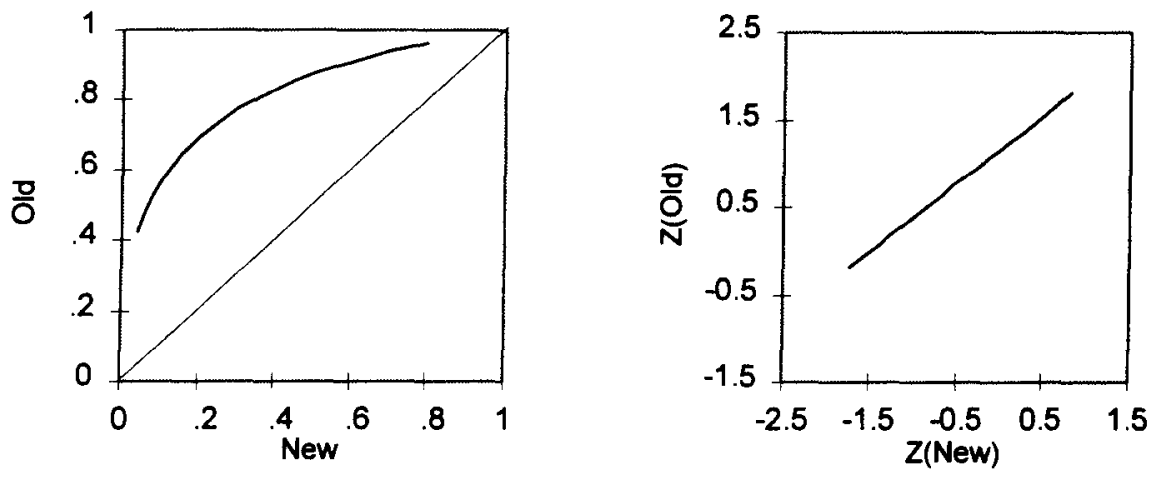

d) Recollection
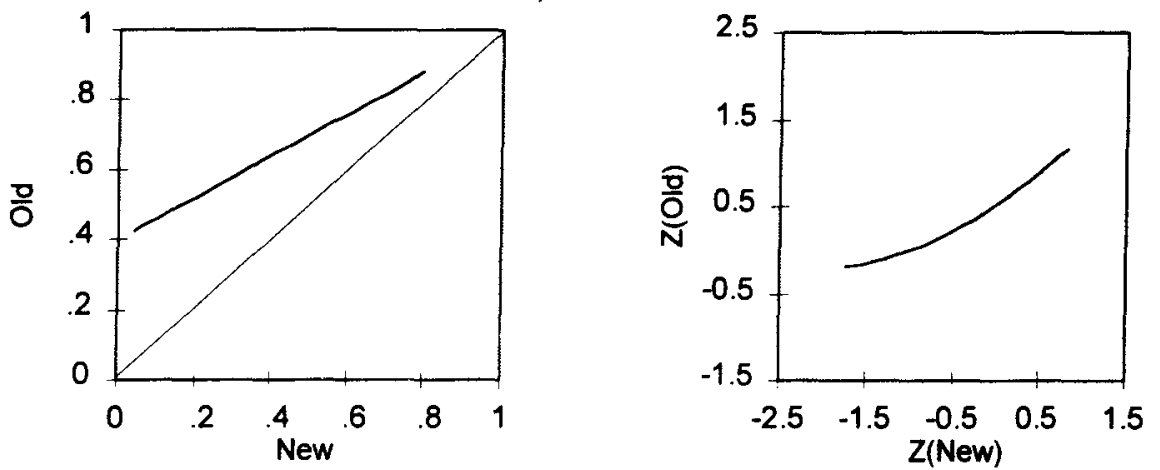

Figure 1. ROCs generated by (a) the equal-variance signal detection model (familiarity), (b) the dual-process signal detection model (familiarity and recollection), (c) the unequal-variance signal-detection model, and (d) the high-threshold model (recollection). ROCs are plotted in probability space and $z$-space in the left and right panels, respectively. 
timate the slope of the function (see the ROCs in the right panels of Figure 1). A perfectly symmetrical ROC, like that in Figure la, will have a slope of 1.0 in $z$-space, and asymmetrical curves like that in Figures $1 \mathrm{~b}$ and $1 \mathrm{c}$ will have a slope of less than 1.0. Recognition memory ROCs typically have slopes around .8 , but they can vary from .3 to close to 1.0. The $z$-ROC is often used to measure memory sensitivity (i.e., $d^{\prime}=$ intercept/slope).

The symmetry of the observed ROCs tends to vary systematically. In some cases, the ROCs become more asymmetrical as performance increases. This would be reflected in $z$-space as an increase in the intercept accompanied by a decrease in slope. Variables that lead to an increase in intercept and a decrease in slope include study-test lag (Donaldson \& Murdock, 1968), word frequency (Glanzer \& Adams, 1990; Ratcliff, McKoon, \& Tindall, 1994), word concreteness, and word decoding (Glanzer \& Adams, 1990). Changing list length tends to have similar effects (Gronlund \& Elam, 1994; Ratcliff, et al. 1994; Yonelinas, 1994; note that in the Ratcliff et al. [1994] experiment and in one of the experiments in Gronlund \& Elam [1994], the slope did not change significantly).

Other manipulations increase performance but do not influence the symmetry of the function. For example, increasing study duration increases the intercept but does not have any noticeable effect on $z$-ROC slope. Ratcliff et al. (1992), Ratcliff et al. (1994), and Yonelinas (1994) found that increasing study time led to an increase in the intercept, but the slope remained constant at approximately .8. Similarly, Egan (1958) reported that performance was greater for items presented twice at study than for those presented once, but that the slope for both curves was constant at approximately .7. Finally, Yonelinas and Jacoby (1995) examined the effects of changing the size of random shapes between study and test on recognition memory and found that changing size was associated with a decrease in intercept but that slope remained constant at approximately .8 .

In summary, the recognition ROCs are curvilinear and asymmetrical. Moreover, the symmetry (i.e., slope) changes across some manipulations but not across others. This pattern of results proves to be difficult for current memory models to account for (see Ratcliff et al., 1992). For example, TODAM (Murdock, 1982) predicts that as performance increases, the ROC slope will remain relatively constant and very close to 1.0 . In contrast, SAM (Gillund \& Shiffrin, 1984) and MINERVA2 (Hintzman, 1986) predict that as performance increases, the slope should decrease. None of these models can account for the full range of observed ROCs. However, the results are in agreement with a dual-process model. I will first describe the evidence in favor of dual-process theories in general and then describe the dual-process model that accounts for the recognition ROC data.

\section{Dual-Process Theories}

Dual-process theories of memory postulate that two qualitatively different processes or systems underlie memory judgments (e.g., Atkinson \& Juola, 1974; Jacoby \&
Dallas, 1981; Mandler, 1980). Evidence in support of dualprocess theories thus comes from observed dissociations between performance on direct and on indirect tests of memory (for reviews, see Richardson-Klavehn \& Bjork, 1988; Roediger \& McDermott, 1993). Similar evidence comes from recognition memory studies, in which dissociations are observed between judgments based on the assessment of familiarity and those based on the recollection of qualitative information about the study event. A dramatic example of such a dissociation comes from the study of amnesic patients, who are able to make recognition judgments based on assessments of familiarity, but are impaired on recognition judgments requiring recollection (e.g., Huppert \& Piercy, 1976; Verfaellie \& Treadwell, 1993). Similarly, aging leads to a selective deficit in recollection (Jennings \& Jacoby, 1993). Moreover, increasing memory load (i.e., list length) interferes primarily with recollection (Yonelinas, 1994; Yonelinas \& Jacoby, 1994), and recognition judgments based on recollection tend to be slower than those based on familiarity (Atkinson \& Juola, 1974; Mandler \& Boeck, 1974; Toth, 1996; Yonelinas \& Jacoby, 1994, 1996).

The two processes have also been found to differ in terms of their phenomenological and electrophysiological correlates. Recollection is accompanied by the awareness of qualitative information about the study event. Familiarity, on the other hand, is not associated with this type of awareness but rather reflects a sense of oldness or fluency. Tulving (1985) developed a procedure in which subjects were asked to introspect about their recognition responses and respond remember whenever they recollected qualitative information about the study event in which the test item was previously studied, and know whenever they recognized an item on the basis of its being familiar and not recollected. Subjects have been found to report on these processes systematically, and the two types of reports are dissociated across numerous variables (see Gardiner, 1988; Gardiner \& Java, 1993; Tulving, 1985; however, see Donaldson, 1996; Yonelinas \& Jacoby, 1995). Recent electrophysiological studies have shown that recollected test items are accompanied by evoked related potentials (ERPs) that are distinct from those evoked by items accepted on the basis of familiarity (Düzel, Yonelinas, Mangun, Heinze, \& Tulving, 1997; Smith, 1993; Wilding \& Rugg, 1994).

Most important for the present study is that familiarity appears to reflect the assessment of a continuous dimension, whereas recollection reflects a relatively discrete retrieval process. Yonelinas (1994) used the process dissociation procedure (Jacoby, 1991) to estimate the contribution of recollection and familiarity to recognition memory performance and found that familiarity increased gradually as response criterion was relaxed. However, judgments based on recollection (i.e., subjects could recollect the source of the memory) led to very highconfidence recognition responses that remained relatively constant as a function of response criterion. On the basis of the ROC results, a dual-process signal detection model was developed. 


\section{The Dual-Process Signal Detection Model}

The dual-process signal detection model assumes that familiarity is well described by signal detection theory (for discussions of signal detection theory, see Banks, 1970; Green \& Swets, 1966; Macmillan \& Creelman, 1991; Murdock, 1965; Swets, Tanner, \& Birdsall, 1961; Wickelgren \& Norman, 1966). The idea is that new items will vary in their preexperimental familiarity in such a way that the familiarity values form a normal distribution. Studying items will increase the familiarity of those items, so that, on the average, they will be more familiar than the new items. However, the old and new item distributions overlap, so subjects must set some response criterion and accept the most familiar items as old. Subjects can vary their response criterion on familiarity and increase or decrease their hits and false alarms.

Subjects, however, are not limited to assessments of familiarity. If they can recollect information about the study event, they can use this as a basis for recognition judgments. Recollection is assumed to be a discrete retrieval process, such that the subject either retrieves or fails to retrieve a specific aspect of a study event. Subjects, of course, can recollect different aspects of the study event, such as information about the physical context or what they thought about when the item was studied. However, when recollection occurs, it is expected to lead to a highconfidence recognition judgment. The simplest way of describing such a process is to treat it as a threshold process, and thus recollection can be measured as a simple probability.

Recollection and familiarity are assumed to serve as two independent bases for recognition judgments (for a discussion of the independence assumption, see Cowan \& Stadler, 1996; Jacoby, Toth, \& Yonelinas, 1993; Jacoby, Toth, Yonelinas, \& Debner, 1994; Jacoby, Yonelinas, \& Jennings, 1997; Joordens \& Merikle, 1993). Thus, the probability of recognizing an old item is equal to the probability that it will be recollected $\left(R_{\mathrm{o}}\right)$, plus the probability that its familiarity exceeds the response criterion $\left(F_{\mathrm{o}}\right)$, minus the probability that the item is recollected and familiar:

$$
P \text { ("yes" } \mid \text { old })=R_{\mathrm{o}}+F_{\mathrm{o}}-R_{\mathrm{o}} F_{\mathrm{o}} .
$$

The probability that a new item will be incorrectly accepted as old will be equal to the probability that its familiarity exceeds the response criterion:

$$
P(\text { "yes" } \mid \text { new })=F_{\text {n }} \text {. }
$$

Equations 1 and 2 can be combined to form a single equation that relates the hit rate to the false alarm rate. Given Equations 1 and 2,

$P$ ("yes"| old $)-P($ "yes"| $\mid$ new $)=R_{\mathrm{o}}+F_{\mathrm{o}}-R_{\mathrm{o}} F_{\mathrm{o}}-F_{\mathrm{n}}$;

thus,

$$
\begin{aligned}
P(\text { "yes" } \mid \text { old })= & R_{\mathrm{o}}+F_{\mathrm{o}}-R_{\mathrm{o}} F_{\mathrm{o}} \\
& +P(\text { ("yes" } \mid \text { new })-F_{\mathrm{n}} .
\end{aligned}
$$

Familiarity is assumed to reflect a signal detection model of the type that underlies standard $d^{\prime}$ tables (e.g., Elliott, 1964), and thus the new and old item distributions are assumed to be normal and to have the same variance. This means that $F_{\mathrm{o}}$ and $F_{\mathrm{n}}$ will be a function of $d^{\prime}$ (the distance between the means of the means of the old and new item distributions) and $c$ (the response criterion). If the familiarity distributions are normal and of equal variance, then $F_{0}=\Phi\left(d^{\prime} / 2-c\right)$ and $F_{0}=\Phi$ $\left(-d^{\prime} / 2-c\right)$. These functions represent the proportion of the target and lure distributions that exceed the response criterion $(c)$, given that the distance between the means of the two normal distributions is $d^{\prime}$.

If the model is correct, memory performance (e.g., the ROC) will be highly constrained. If recognition judgments are based on familiarity alone (i.e., $R_{\mathrm{o}}=0$ ), the ROC will look like Figure 1a. The function increases gradually toward 1,1 , and it forms a curvilinear function that is symmetrical along the negative diagonal. In contrast, if recognition judgments are based on recollection alone (i.e., $d^{\prime}=0.0$ ), the ROC will look like Figure 1d. Recollection will put a lower limit on the hit rate, and the function will increase in a linear fashion toward 1,1. If recollection and familiarity contribute to performance, the predicted ROC will look like Figure $1 \mathrm{~b}$. The ROC is curvilinear and increases toward 1,1 , but it is asymmetrical along the negative diagonal.

\section{The Dual-Process Model and Recognition Memory ROCs}

The dual-process model is in agreement with the existing recognition ROC data, and recent studies have shown that there is a direct relationship between the shape of the ROC and the measured contribution of recollection and familiarity. The model predicts that because recollection and familiarity contribute to performance, the ROCs should be curvilinear and asymmetrical, as is usually the case. Familiarity leads to a symmetrical ROC, but recollection will increase the hit rate and push the ROC up, resulting in an asymmetrical ROC (i.e., the slope of $z$-ROC will be less than 1.0). Moreover, as long as familiarity remains relatively constant, then increasing recollection will lead the ROC to become less symmetrical. Thus the model can account for the finding that an increase in overall performance can be associated with a decrease in the slope of the $z$-ROC. If, however, both recollection and familiarity increase together, overall performance will increase but the slope of the $z$-ROC will remain relatively constant. Increasing recollection will tend to force the ROC to become more asymmetrical; increasing familiarity, however, will tend to force the ROC to become more symmetrical (for a detailed description of the model's predictions, see Yonelinas, 1994). Thus the model can account for the observation that ROCs are often asymmetrical and that increases in performance can be accompanied either by a decrease in symmetry or by no change in symmetry. 
Further support for the model comes from studies showing a direct relationship between the processes underlying recognition judgments and the shape of the ROCs. For example, when recollection and familiarity were estimated with the process dissociation procedure, shortening list length was found to lead to an increase in recollection, but familiarity was left relatively unaffected (Yonelinas, 1994). As the model predicted, the increase in recollection associated with shortening the list length led to an increase in overall performance and a decrease in the slope of the $z$-ROC. In contrast, in a subsequent experiment, an increase in study duration was found to increase both recollection and familiarity, and this led to the predicted increase in performance accompanied by no change in the slope of the $z$-ROC. Finally, the model was found to provide accurate quantitative predictions for the slopes and intercepts of ROCs on the basis of simple yes/no responses.

In a related study, subjects were asked to report when they could recollect having seen items in the study list (Yonelinas \& Jacoby, 1995). Changing the size of random geometric shapes between study and test led to a decrease in both recollection and familiarity. As the dualprocess model predicted, an ROC analysis showed that as overall performance decreased, the slope remained constant. Thus, not only can the model produce the observed pattern of ROCs, but it accurately describes the relationship between the shape of the ROC and the estimated contribution of recollection and familiarity.

\section{Can the Recognition Data Be Accounted for Without Introducing Recollection?}

An alternative way of accounting for the ROC data is to use the standard signal detection model but to assume that the variance of the old-item distribution can vary independently of memory sensitivity $\left(d^{\prime}\right)$. If the old-item variance is greater than the new-item variance, the ROC will become asymmetrical, and the slope of the $z$-ROC will drop below 1.0. This unequal-variance signal detection model can thus account for any intercept $X$ slope relationship, because it has one free parameter for sensitivity and one parameter that influences slope. An example of the predicted ROC for the model is presented in Figure $1 \mathrm{c}$. Note that this model does not make predictions regarding the relationship between the shape of the ROC and recollection and familiarity, but it can be used to describe the ROC data.

A comparison of Figures $1 \mathrm{~b}$ and $1 \mathrm{c}$ shows that the unequal-variance signal detection model can produce an ROC that looks very much like that predicted by the dual-process model. However, there are subtle differences between the two models. Because the dual-process model assumes that there is a threshold process (i.e., recollection) that contributes to performance, it predicts ROCs that are slightly flatter than those predicted by a signal detection process alone. Although the differences are small, they are slightly more noticeable when one examines the $z$-ROCs; the dual-process model predicts a slight $\mathrm{U}$-shaped
$z$-ROC in comparison with the unequal-variance signal detection model, which predicts a straight line.

Previous studies of recognition memory have shown that discriminating between these two models in recognition is quite difficult (Yonelinas, 1994; Yonelinas, Dobbins, Szymanski, Dhaliwal, \& King, 1996). However, there are conditions under which it is possible to discriminate between the models. The difference between the models should become quite pronounced under conditions in which the contribution of familiarity is very small and the contribution of recollection is large. The dualprocess model predicts that in a task that relies solely on recollection, the ROCs should be linear. When the ROC is plotted in $z$-space, the function should exhibit a noticeable U-shape (see, e.g., Figure 1d). The unequal-variance signal detection model, on the other hand, predicts a curvilinear ROC and a $z$-ROC that should always be linear. The present experiments were designed to explore the possibility that tests of associative recognition rely primarily on recollection, and that ROCs for this task will be linear (and thus the $z$-ROCs should be U-shaped). The ROC analysis should provide a way of discriminating between the dual-process model and the unequal-variance signal detection model.

\section{Tests of Item and Associative Information}

In all of the previously mentioned ROC studies, memory for item information was tested. That is, subjects judged whether an item had been presented earlier. In the present study, the dual-process model was tested by examining ROCs for recognition of associative information. In tests of associative recognition, subjects must remember which items were paired together in a previous study list. Typically, subjects study a list of word pairs followed by a recognition test containing intact and rearranged pairs. The subject's task is to respond positively to intact pairs and negatively to rearranged pairs.

Recognition memory for associative and item information has been found to differ in several ways. Gronlund and Ratcliff (1989) found, for example, that the information required in order to make item judgments was available earlier than that required in order to make associative judgments. Moreover, Hockley (1991) found that, as the number of items between study and test was increased, memory for item information decreased much more rapidly than memory for associative information. As well, in recognition memory there is an advantage for low- over high-frequency words, yet in tests of associative recognition, there is either no word frequency effect (Hockley, 1994) or an advantage for high- over lowfrequency items (Clark, 1992; Clark \& Shiffrin, 1992). Finally, in indirect tests of memory, amnesics exhibit disproportionate deficits in memory for associative information. Although amnesics can show memory for words and related word pairs (for a review, see Richardson-Klavehn \& Bjork, 1988), severe amnesics exhibit a marked reduction in indirect memory performance for pairs of unrelated words (see, e.g., Schacter \& Graf, 1986). 
Why is item recognition dissociated from associative recognition? One possibility that is explored in the present study is that the two types of tasks rely differentially on recollection and familiarity (for related suggestions, see Anderson, 1974; Clark, 1992; Clark \& Gronlund, 1996; Hockley, 1992; Humphreys, 1978; Wolford, 1971). Tests of item information may rely on recollection as well as assessments of item familiarity. That is, subjects may recollect information about the study event or they may accept the item as old because it is familiar. Tests of associative information, on the other hand, may rely primarily on recollection. Because each of the items in the old and rearranged pairs has been studied, it is likely that both types of pairs will be familiar, and thus familiarity will be of little use when one discriminates between old and new pairs.

The notion that tests of item and associative information rely differentially on recollection and familiarity was tested by examining ROCs. Although a great deal is known about the nature of ROCs for item information, relatively little is known about ROCs for associative information (for an examination of ROCs for intact vs. rearranged pairs in a short-term memory task, however, see Murdock, 1965, 1974). The aim of the present study was to contrast ROCs for item and associative information under standard recognition memory conditions.

If tests of associative information rely on recollection, yet tests of item information rely on both recollection and familiarity, the ROCs for item and associative information should be quite different. The dual-process model predicts that judgments based on recollection and familiarity should produce curvilinear ROCs. In contrast, judgments based on recollection alone should produce linear ROCs. These predictions were assessed in Experiments $1-3$ by examining recognition ROCs for item and associative information. If item judgments rely on recollection and familiarity, the ROC should be curvilinear. In contrast, if associative judgments rely on recollection alone, the ROC should be linear. In $z$-space, the ROCs should exhibit a slight $U$-shape. In item recognition, in which both recollection and familiarity contribute to performance, the curvilinearity (i.e., U-shape) is not expected to be large (see Figure lb); in associative recognition, however, the U-shape should become more noticeable (see Figure 1d).

If, on the other hand, item and associative recognition rely on a signal detection process alone (i.e., an unequalvariance signal detection model), the ROCs should always be curvilinear. This means that in $z$-space, the ROCs should be linear, regardless of the task. Finding a U-shaped $z$-ROC would support the dual-process model and contradict the signal detection model.

\section{Recollecting New Pairs as New}

In addition to contrasting the shape of the item and associative ROCs, the present study is intended to test one of the more intuitively appealing aspects of threshold theories. The notion is that subjects may be able to recollect that some test pairs (or test items) were not in the study list. That is, subjects may sometimes correctly call a rearranged pair of words new because they recollect that the words were not paired together in the study list. For example, imagine that the rearranged pair $\mathrm{A}-\mathrm{F}$ was presented at test, and the subject could recollect that $\mathrm{A}$ was paired with B at study. If each study item was paired with only one other item, the subject could use recollection to conclude that A-F was not in the study list. In this way, subjects could recollect that rearranged pairs were new and avoid false alarming to those pairs.

Subjects might also recollect that new items were new. For example, after studying a short list of words, one would likely not false alarm to one's own name if it appeared in the test list. However, in typical lab tests of recognition, differences between old and new items are strictly controlled, and words are most often selected from a homogeneous pool. Thus, recollection of new items as new may not play a large role in standard recognition memory tests. I will return later to evidence in the present studies suggesting that subjects do not often recollect new items as new.

The possibility that subjects can recollect new pairs as new cannot be accounted for by the dual-process model described earlier. To do so, it is necessary to generalize the model by introducing a term to represent the probability that a new pair is recollected as new. The probability of recognizing an old pair will remain the same as the original dual-process equation (Equation 1); the probability of accepting a new pair as old, however, will be equal to the probability that it is sufficiently familiar and that it was not recollected as new $\left(1-R_{\mathrm{n}}\right)$ :

$$
P \text { ("yes" } \mid \text { new })=F_{\mathrm{n}}\left(1-R_{\mathrm{n}}\right) .
$$

The notion behind the equation is that if a subject does recollect the pair as new, the subject would not false alarm to it. By combining Equations 1 and 4, memory performance can be described as:

$$
\begin{aligned}
P(\text { "yes" } \mid \text { old })= & R_{\mathrm{o}}+F_{\mathrm{o}}-R_{\mathrm{o}} F_{\mathrm{o}} \\
& +P(\text { “yes" } \mid \text { new })-F_{\mathrm{n}}\left(1-R_{\mathrm{n}}\right) .
\end{aligned}
$$

The equation is somewhat unwieldy, but if familiarity does not contribute to recognition performance, $F_{0}=F_{\mathrm{n}}$ and the terms can be replaced by a guessing term $(\mathrm{G})$. Doing so, the model reduces to a simple linear equation:

$P$ ("yes"| old $)=R_{0}-G\left(R_{\mathrm{o}}-R_{\mathrm{n}}\right)+P($ "yes" $\mid$ new $)$.

The equation reflects a threshold model in which the $y$-intercept is equal to $R_{0}$ and the upper $x$-intercept is equal to $1-R_{\mathrm{n}}$. If the model is correct, associative ROCs should be a straight line, and the line's intercepts can be used as measures of $R_{0}$ and $R_{\mathrm{n}}$.

If subjects cannot recollect that new pairs are new, $R_{\mathrm{n}}$ should be zero, and the ROC should approach the 1,1 intercept (as in Figure 1d). In contrast, if subjects can recollect that new pairs are new, $R_{\mathrm{n}}$ should be greater than zero, and the ROC should approach some point less than 1.0 on the upper $x$-axis. That is, if subjects recollect that $20 \%$ of the new pairs are new, their false alarm rate should 
not exceed $80 \%$. Thus, as the false alarm rate increases, the ROC should approach the upper $x$-axis at 8 .

If the threshold notion is correct, it should be possible to systematically vary $R_{\mathrm{n}}$. To test this notion, the likelihood that subjects would recollect rearranged pairs as new was varied across experiments. Experiment 1 was designed to make it difficult to recollect rearranged pairs as new. Word pairs were studied in a continuous list such that each word was paired with two other words. In this way, subjects had to recollect both of the words that were earlier paired with one of the target words in order to recollect that the pair was new. Recollecting only one of the previously paired words would not be sufficient to conclude that the target pair was not studied. $R_{\mathrm{n}}$ was expected to be quite low under these conditions. In contrast, Experiments 2 and 3 were designed to promote recollection that rearranged pairs were new. Each word was paired with only one other word at the time of study. In this way, subjects could recollect that a pair was new if they could recollect that one of the target words had previously been paired with another word.

In summary, ROCs for item and associative information were examined in three experiments. The dualprocess model predicts that the ROCs for item information will be curvilinear. If associative judgments are based on recollection alone, however, the associative ROC should be linear. Moreover, in Experiment 1, in which recollecting rearranged pairs as new was made difficult, $R_{\mathrm{n}}$ should be low and the ROC should approach the upper $x$-axis at 1.0. In contrast, in Experiments 2 and 3, in which recollecting that rearranged pairs are new is less difficult, $R_{\mathrm{n}}$ should be greater, and the function should intersect the upper $x$-axis at some point less than 1.0.

\section{EXPERIMENT 1}

\section{Method}

Subjects and Materials. Twenty undergraduates participated in the experiment for a credit in an introductory psychology course. Three hundred and four words were randomly selected from the Toronto word pool for each subject.

Design and Procedure. Materials were presented and responses collected on an IBM-compatible computer. The character size of the stimuli was approximately $5 \times 5 \mathrm{~mm}$. The stimuli were presented in uppercase letters in the center of the screen. In the study phase, a list of words was presented two words at a time. For example, the study phase began with the first and second words presented on the screen side by side. The next pair of words consisted of the second and third words from the list. In this way, each of the critical words was paired with two others. Each word pair was presented for $4 \mathrm{sec}$. The study list consisted of 300 critical words and four buffer words.

The study list was divided in half. After the first half was presented there was a 1 -min break. The first and last words in each half were buffer words and were not tested. The subjects were instructed to remember the words for a later recognition memory test. They were informed that they would be required to remember which words were presented and which words were paired together.

Immediately after the study phase, the subjects received a recognition memory test for item and associative information. A random selection of items and pairs of items was presented. The subjects were told that if one word appeared on the screen they were to de- cide whether the word had or had not been presented in the study list. If a pair of words appeared, they were to decide whether those two words had or had not been paired together in the study list. The test list consisted of 60 intact word pairs (i.e., items that had been studied together) and 60 rearranged word pairs (i.e., items that had not been studied together), and 60 single words from the study list and 60 single new words. The pairs and items were presented in a random order, and the order of words within the pairs (i.e., left or right side) was determined randomly at test. No words were tested in two different pairs or both as an item and as part of a pair.

The subjects were instructed to make their responses on a 6-point confidence scale from sure no (1) to sure yes (6). The subjects were instructed to try to spread their responses across the scale so that they used all the response keys. The experimental session took approximately $45 \mathrm{~min}$ to complete. The significance level for all statistical tests was $p<.05$.

\section{Results and Discussion}

Figure 2 presents the observed ROCs for item and associative (pair) recognition for Experiment 1, along with functions generated by the dual-process model. For the item judgments, the probability of responding yes to old items is plotted against the probability of responding yes to new items. Performance is plotted as a function of response confidence such that the left-most point reflects only the most confident yes responses (i.e., items eliciting a " 6 " response) and the second point includes the items eliciting a " 5 " or a " 6 " response. Thus the 6-point confidence scale produced 5 points on the ROC. The associative ROC was calculated in the same way. The proportion of old pairs that correctly led to a yes response was plotted against the proportion of rearranged pairs leading to a yes response.

Examination of Figure 2 shows that the ROC for the item judgments exhibited a systematic deviation from linearity (i.e., an inverted U-shape), as was expected if familiarity contributed to performance. In contrast, the associative ROC was relatively flat and was fit well by a straight line, which was expected if associative judgments were based on recollection alone. Moreover, the associative ROC approached the 1,1 intercept, suggesting that subjects did not often recollect new pairs as new.

To assess the linearity of the ROCs further, linear and quadratic regressions were conducted to determine whether the ROCs exhibited a significant linear trend, and to test whether there was a significant improvement in the fit of the regression equation when a nonlinear (quadratic) component was introduced. The $R^{2}$ values associated with the linear and the nonlinear equations are presented in Table 1. For the item recognition ROC, there was a significant linear component $\left[F(1,3)=39.24, M S_{\mathrm{e}}=\right.$ $.003]$. However, introducing the quadratic led to a significant improvement in the fit of the equation $[F(1,2)=$ $\left.189.97, M S_{\mathrm{e}}=.001\right]$, showing that the item recognition ROC was nonlinear. For the associative recognition ROC, there was a significant linear component $[F(1,3)=$ $\left.1064.77, M S_{\mathrm{e}}=.0003\right]$. Moreover, introducing the quadratic component did not lead to a significant improvement in the fit of the equation $\left[F(1,2)=1.00, M S_{\mathrm{e}}=\right.$ $.002]$, showing that the associative ROC was described 

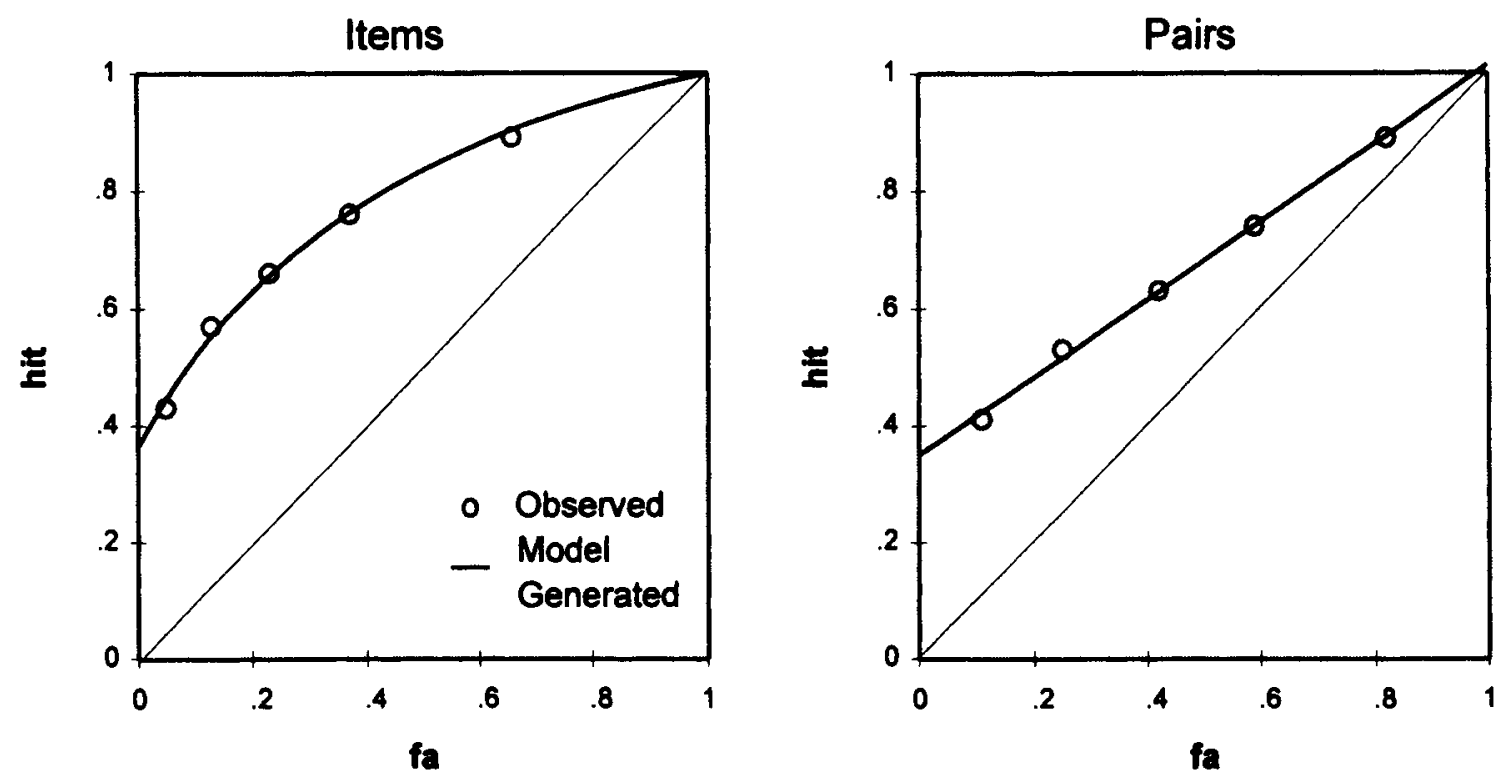

Figure 2. ROCs for item and associative (pairs) recognition plotted along with the functions generated by the dual-process model for Experiment 1.

well by a linear function. Thus the linearity analysis supports the conclusions drawn from the visual examination of the ROCs; the item ROC was curvilinear and the associative ROC was linear.

To determine whether the ROCs could be described by a pure signal detection model, the functions were plotted in $z$-space (Figure 3 ) and a linear trend analysis was conducted. If the unequal-variance signal detection model is correct, the $z$-ROCs should be straight lines. Examination of Figure 3 shows that the item $z$-ROC was close to linear, but the associative $z$-ROC was noticeably U-shaped. Linear trend analyses supported these observations. The $R^{2}$ values from the linearity analysis are presented in Table 1. For the item $z$-ROC, there was a significant linear component $\left[F(1,3)=3,767.33, M S_{\mathrm{e}}=.0003\right]$, and introducing the quadratic did not lead to a significant im-

Table 1

$R^{2}$ Values for the Linear and the Linear + Quadratic Equations for the Average Item and Associative ROCs and $z$-ROCs in Experiments 1, 2, and 3

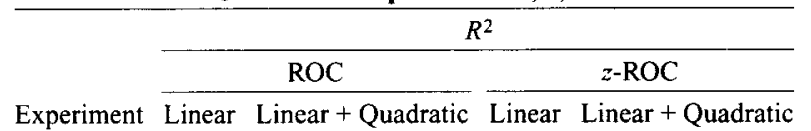

\begin{tabular}{lcccc}
\multicolumn{5}{c}{ Item Information } \\
1 & .929 & $.992 \dagger$ & .999 & .999 \\
2 & .889 & $.995 \dagger$ & .997 & .999 \\
3 & .959 & $.996^{*}$ & .994 & .999 \\
& \multicolumn{4}{c}{ Associative Information } \\
1 & .997 & .998 & .986 & $.999^{*}$ \\
2 & .968 & .993 & .993 & .998 \\
3 & .997 & .999 & .947 & $.999^{*}$ \\
\hline
\end{tabular}

Note-Symbols denote conditions in which the quadratic component led to a significant improvement in $R^{2}$ values over the linear model $\left({ }^{*} p<.05,{ }^{+} p<.01\right)$. provement in the fit of the equation $(F<1)$, suggesting that the $z$-ROC was linear. The slope of the recognition $z-\mathrm{ROC}$ was .68 , which is typical of other recognition memory studies. For the associative recognition $z$-ROC, however, there was a significant linear component $[F(1,3)=$ 216.27, $\left.M S_{\mathrm{e}}=.006\right]$, and introducing the quadratic component led to a significant improvement in the fit of the equation $\left[F(1,2)=26.00, M S_{\mathrm{e}}=.001\right]$, indicating that the $z$-ROC was not linear. The U-shaped $z$-ROC observed for the associative ROC shows that the unequalvariance signal detection model was in conflict with the data and is in agreement with the notion that associative recognition relies primarily on a threshold recollection process.

Although the linear trend analysis provides a simple way of assessing the linearity of the ROCs, two points about the analysis should be made. First, there may be nonlinear functions other than the quadratic that fit the ROCs better. However, for the present data sets (Experiments 1-3), the quadratic was found to provide a very good fit to the ROCs (the average $R^{2}$ for item ROCs was $.995)$, suggesting that the quadratic does provide a reasonable way of assessing the curvilinearity of the observed functions. Second, the regression analysis assumes that the points in each function are independent. This assumption was not met in the present experiments, because the ROCs are cumulated across response confidence. However, the cumulative method used here has been found to lead to ROCs that are similar in shape to those observed when noncumulative methods are used (see Ratcliff et al., 1992). Most importantly, the results of the linear trend analysis converge with the conclusions drawn from the visual examination of the ROCs.

Could the results of the ROC analysis have been influenced by averaging? Although averaging across sub- 


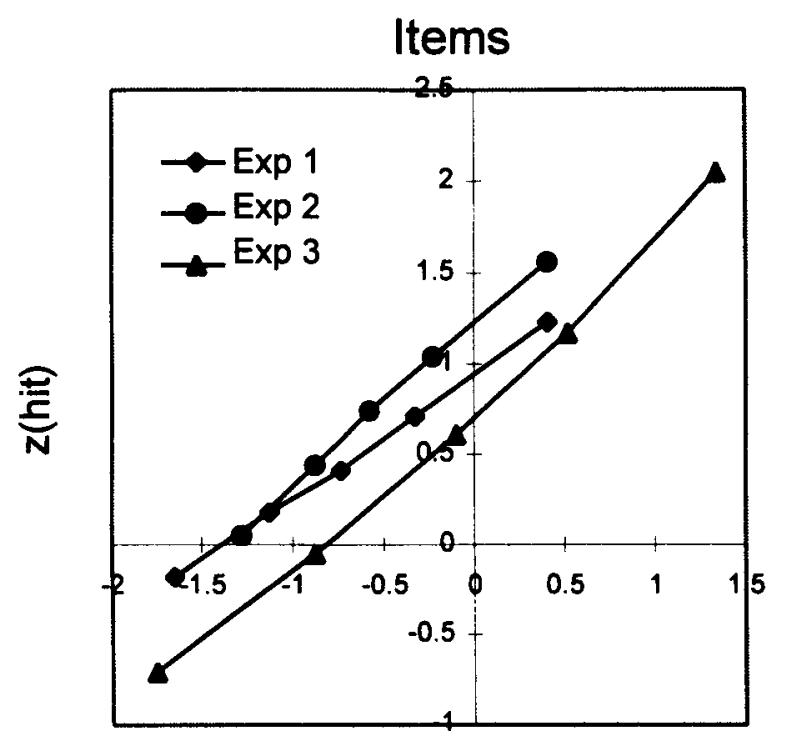

$z(\mathrm{fa})$

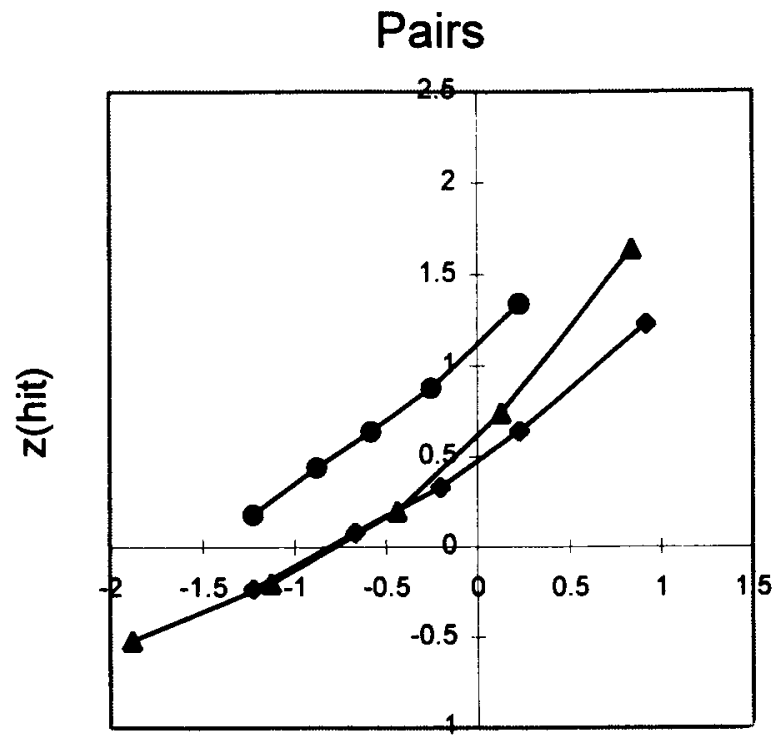

$z(\mathrm{fa})$

Figure 3. $z$-ROCs for item and associative information for Experiments 1, 2, and 3.

jects or items could influence the shape of the ROCs, it is unlikely to have produced the differences that were observed between the item and associative ROCs. Given that ROCs were averaged in the same way for item and associative information, any artifactual effect of averaging would be expected to influence the ROCs in the same way, making the two ROCs more similar. In any case, the effect of averaging was examined by plotting the ROCs as a function of test position and subject. The linear and nonlinear aspects of the associative and item ROCs were not found to differ as a function of test position or subject. Although the individual subject ROCs were based on relatively few observations, 18 of the 20 subjects exhibited the same pattern of results as that seen in the average ROCs; the associative ROC was relatively flat in comparison with the item ROC. Moreover, in Experiment 3, a larger number of observations were collected for each subject and an analysis of individual ROCs showed that averaging across subjects was not responsible for the observed difference between item and associative ROCs.

To further assess the dual-process model, and to determine whether subjects did recollect new pairs as new, the model was fit to the observed ROCs. Figure 2 presents the model-generated functions along with the observed ROCs for item and associative recognition for Experiment 1 . The model was fit to the data by minimizing the sum of squared errors between the predicted function and the observed ROC points. The procedure is analogous to conducting a linear regression to determine the slope and intercept of a line; in this case, however, the function could be nonlinear and the estimated parameters reflected recollection and familiarity. Because the functions could be curvilinear and the points in each function were free to vary in the $y$ - and $x$-dimensions (i.e.. hits and false alarms), the sum of squared error term included variation in hits and false alarms. The item ROC was fit to the standard dual-process model that has been used in previous recognition studies (Equation 3). The associative ROC was fit to the generalized dual-process model (Equation 6). However, because familiarity was not expected to contribute to performance, and because the linearity analysis suggested that a linear model provided an accurate account of performance, the familiarity component was not used for the associative ROCs.

An examination of Figure 2 shows that the dual-process model provided an accurate account of memory performance. For both item and associative recognition, the observed ROC points fell very close to the model-generated functions.

The parameter estimates derived by fitting the model to the observed ROCs are presented in Table 2. As was expected, the probability that new pairs were recollected as new was quite low (.02). In fact, a single parameter version of the model $\left(R_{\mathrm{o}}=.35\right)$ could not be rejected in favor of the two-parameter model $\left[F(1,3)=2.86, M S_{\mathrm{e}}=\right.$ .0003 ], showing that the contribution of $R_{\mathrm{n}}$ was negligible. Thus the model suggests that subjects did not often recollect rearranged pairs as new.

The results of Experiment 1 showed that the ROC for item judgments was curvilinear, as was expected if performance relied on recollection and familiarity. In contrast, the ROC for associative recognition was found to be linear, as was expected if judgments were based on recollection alone. Although the item ROC was in general agreement with the unequal-variance signal detection model, the U-shape of the associative $z$-ROC showed that the signal-detection model could not account for performance. In contrast, the dual-process model was found to provide an accurate fit for the item and associative ROCs. 
Table 2

Parameter Estimates for Recollection $(R)$ and Familiarity $\left(d^{\prime}\right)$ for Item and Associative ROCs in Experiments 1, 2, and 3

\begin{tabular}{ccccc} 
& \multicolumn{4}{c}{ Condition } \\
\cline { 2 - 5 } Experiment & $R_{\mathrm{o}}$ & $d^{\prime}$ & & \multicolumn{2}{c}{ Associative } \\
\cline { 2 - 5 } & .35 & 0.66 & .35 & $R_{\mathrm{o}}$ \\
\hline 1 & .14 & 1.17 & .52 & .31 \\
2 & .14 & 0.58 & .30 & .16 \\
\hline 3 & &
\end{tabular}

Note-For item recognition, $d^{\prime}$ refers to the distance between the means of the old and new item familiarity distributions, and $R_{0}$ refers to the probability of recollecting an old item. For associative recognition, $R_{\mathrm{o}}$ refers to the probability of recollecting an old pair as old, and $R_{\mathrm{n}}$ refers to the probability of recollecting a new pair as new (i.e., recollecting that the items were not paired together at study).

Furthermore, as was expected, the probability of recollecting that a rearranged pair was new was found to be quite low, suggesting that subjects were generally unable to recollect that rearranged pairs were not in the study list.

\section{EXPERIMENT 2}

In Experiment 2, item and associative ROCs were examined again; the threshold notion was tested further, however, by making it easier for subjects to recollect that new pairs were new. Several changes were made to the procedure to increase the likelihood that subjects would recollect that rearranged pairs were new. Unlike in the previous experiment, each item was paired with only one other word in the study list. In this way, subjects could recollect that a pair was rearranged if they could recollect that either of the target items was previously paired with another item. Furthermore, a repeated study-test procedure was used, in which each study list contained 24 word pairs and the type of test was varied between lists. Because the associative judgments were blocked, and because the study lists contained only 24 word pairs, it was expected that subjects would be more likely to try to retrieve the words previously paired with the target items. It was expected that making it easier for subjects to recollect that rearranged pairs were not in the study list would increase $R_{\mathrm{n}}$. Thus, as the hit rate increased, the false alarm rate should not approach 1.0, as in the previous experiment, but should approach some value considerably less that 1.0 .

\section{Method}

Subjects and Materials. Twenty subjects from the same pool as that for Experiment 1 participated in Experiment 2. Eight hundred and forty words were randomly selected from the Toronto word pool for each subject.

Design and Procedure. The design and procedure were similar to those for the previous experiment, with the following changes. The subjects were presented with 15 study-test blocks. In each block, the subjects were presented with a list of 24 word pairs. No item appeared in more than one pair, and new items were chosen for each study-test block. Each pair was studied for $3 \mathrm{sec}$. After the presentation of each study list, the subjects received a recognition test either for items or for pairs. For 10 of the 15 blocks, they were tested for associative information, and for the remaining 5 blocks, they were tested for item information. The blocks were presented in a random order such that subjects did not know what type of test they would receive until the test began. For the item tests, the subjects were presented with 24 old and 24 new items, rearranged in a random order. For the associative tests, the subjects were presented with 12 intact word pairs and 12 rearranged word pairs. As in the previous experiment, the subjects responded on a 6-point confidence scale.

\section{Results and Discussion}

Figure 4 presents the observed ROCs for item and associative recognition, along with the model-generated functions for Experiment 2. As in the previous experiment, the item ROC was curvilinear, and the associative ROC was fit reasonably well by a linear function. However, a close examination of the associative ROC suggests that it exhibited a slight inverted U-shape. Unlike in Experiment 1 , the associative ROC did not approach the 1,1 intercept, but approached the upper $x$-axis at a point considerably less than 1.0 , as was expected if subjects were able to recollect that rearranged pairs were new.

To assess the linearity of the ROCs further, linear and quadratic regressions were conducted $\left(R^{2}\right.$ values are presented in Table 1). For the item recognition ROC, there was a significant linear component $\left[F(1,3)=24.09, M S_{\mathrm{e}}=\right.$ $.004]$, but there was a significant improvement in the fit of the equation when the quadratic was introduced $[F(2,3)=$ $\left.42.40, M S_{\mathrm{e}}=.005\right]$, showing that the item recognition ROC was nonlinear. For the associative recognition ROC, there was a significant linear component $[F(1,3)=90.75$, $\left.M S_{\mathrm{e}}=.001\right]$, and introducing the quadratic component did not lead to a significant improvement in the fit of the equation $\left[F(2,3)=7.143, M S_{\mathrm{e}}=.007\right]$. Thus, the linearity analysis suggests that the slight curve that was seen in the associative ROC was not sufficient to warrant introducing a nonlinear component.

To determine whether the ROCs could be described with a signal detection based model, the functions were plotted in $z$-space (Figure 3). Examination of Figure 3 shows that the item and associative $z$-ROCs were relatively linear. For the item $z$-ROC, there was a significant linear component $\left[F(1,3)=1140.81, M S_{\mathrm{e}}=.001\right]$, and introducing the quadratic did not lead to a significant improvement in the fit of the equation $\left[F(1,2)=8.00, M S_{\mathrm{e}}=\right.$ $.001]$. For the associative recognition $z$-ROC, there was a significant linear component $\left[F(1,3)=483.28, M S_{\mathrm{e}}=\right.$ $.002]$, and introducing the quadratic component did not lead to a significant improvement in the fit of the equation $\left[F(1,2)=5.00, M S_{\mathrm{e}}=.002\right]$. The slopes of the item and associative $z$-ROCs were .89 and .77 , respectively.

The results of the ROC analysis are similar to those of Experiment 1 in showing that the item and associative ROCs were curvilinear and linear, respectively. However, the visual inspection of the associative ROC suggested that it did exhibit a slight curve. Moreover, unlike in Experiment 1, the associative $z$-ROC was not found to be significantly U-shaped, although it did exhibit a slight U-shape. Nonetheless, the nonlinearity in Experiment 2 was not sufficient to reject the unequal-variance signal detection model. Why the U-shape of the associative 

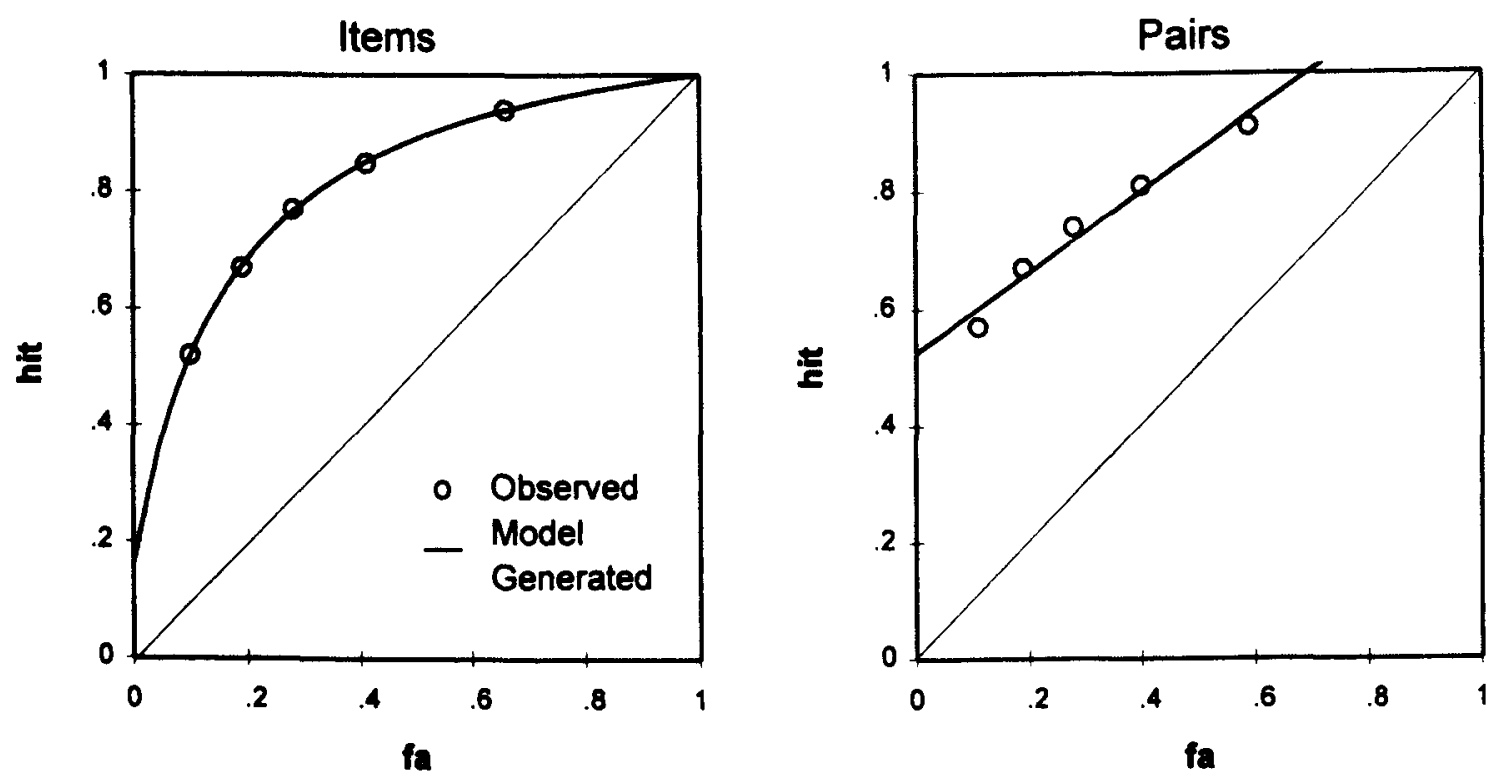

Figure 4. ROCs for item and associative recognition plotted along with the functions generated by the dual-process model for Experiment 2.

$z$-ROC was not significant in the present experiment will be discussed after the presentation of the final experiment.

As in Experiment 1, the dual-process model was fit to the observed ROCs (see Figure 4). The item ROC was fit to Equation 3, and the associative ROC was fit to Equation 6 (with the restriction that familiarity did not contribute to performance). An examination of Figure 4 shows that the dual-process model provided an accurate account of the recognition ROC and a reasonable approximation of the associative ROC. Note that the model would provide a better fit for the associative ROC if the familiarity process was assumed to contribute to performance; the linearity analysis suggested that the function did not deviate significantly from linearity, however, and thus the familiarity component was not utilized.

The parameter estimates are presented in Table 2. For the associative ROC, the parameter estimate for $R_{\mathrm{n}}$ was .31 , representing the probability that new pairs were recollected. As expected, the probability that new pairs were recollected as new was greater than that found in Experiment $1(.02)$. Unlike in Experiment 1 , the singleparameter version of the model $\left(R_{0}=.60\right)$ could be rejected in favor of the two-parameter model $[F(1,3)=$ $\left.22.01, M S_{\mathrm{e}}=.002\right]$, indicating that $R_{\mathrm{n}}$ did contribute to performance.

In sum, the results of Experiment 2 are similar to those of Experiment 1, in that the item recognition ROC was curvilinear and the associative ROC was linear. Moreover, the dual-process model provided a reasonable account of the observed ROCs. However, unlike in Experiment 1, the associative ROC did exhibit a slight inverted U-shape and the associative $z$-ROC was not sufficiently curved to reject the unequal-variance signal detection model. Although the inverted $\mathrm{U}$-shape seen in the associative ROC was not significant, such a curve would be expected if fa- miliarity contributed to performance. Alternatively, the slight curve might simply have reflected measurement error or noise. For this reason, it was decided to conduct another experiment to determine whether the minor deviation from linearity would be replicated. The issue is important, because deviations from linearity may distort estimates of $R_{0}$ and $R_{\mathrm{n}}$, which were estimated with a linear model. A second reason for conducting an additional experiment was to test the possibility that the ROCs in the previous experiments were influenced by averaging across subjects. Although an examination of subject ROCs in Experiments 1 and 2 suggested that the average ROCs were representative of the individual subject ROCs, the subject ROCs were quite noisy because there were so few observations per subject. In Experiment 3, a greater number of observations were collected from each subject in order to examine subject ROCs more closely.

\section{EXPERIMENT 3}

In Experiment 3, each subject completed several experimental sessions, and item and associative ROCs were examined for individual subjects. As in Experiment 2, each word was paired with only one other word at the time of study. Thus subjects were expected to be able to recollect new pairs as new, the associative ROC should approach the upper $x$-axis at a point less that 1.0 , and $R_{\mathrm{n}}$ was expected to be greater than 0.0. As in Experiment 1, the subjects studied a long list of word pairs followed by a test for item and associative information.

\section{Method}

Subjects and Materials. Six psychology graduate students were paid for their participation. The word pool consisted of 900 words from the Toronto word pool plus 1,224 additional words selected 
from Webster's dictionary. The additional words were selected to be approximately equal in frequency and length to those in the original word pool. For each subject, the words were randomly divided into three groups, such that subjects were tested with different words for each of three sessions.

Design and Procedure. The design and procedure were the same as those in Experiment 1 except for the following changes. In the study phase, word pairs were presented one pair at a time at a $3.5-\mathrm{sec}$ rate. No word appeared in more than one study pair. The study list consisted of 300 critical word pairs and 4 buffer pairs. The test list consisted of 200 pairs (half were mixed) and 200 items (half were studied). Each subject participated in three sessions, spread over a 5-day period, with each session taking approximately $45 \mathrm{~min}$ to complete. Prior to testing, each subject participated in at least three sessions that were similar in design to the critical sessions. The significance level for all statistical tests was $p<.05$.

\section{Results and Discussion}

An initial analysis was conducted on the average ROC data. Figure 5 presents the observed ROCs for item and associative recognition averaged across subjects for Experiment 3 , along with the model-generated functions. As in the previous experiments, the ROC for the item judgments exhibited a noticeable inverted U-shape, and the associative ROC was fit well by a straight line. Moreover, as expected, the associative ROC approached the upper $x$-axis at a point less than 1.0.

A linearity analysis supported the conclusion of the visual inspection of the ROCs. The $R^{2}$ values for the item and associative ROCs are presented in Table 2. For the item recognition ROC, there was a significant linear component $\left[F(1,3)=70.37, M S_{\mathrm{e}}=.005\right]$, but the fit of the equation improved significantly when the quadratic was introduced $\left[F(2,3)=18.00, M S_{\mathrm{e}}=.004\right]$, showing that the item recognition $\mathrm{ROC}$ was nonlinear. For the associative recognition $\mathrm{ROC}$, there was a significant linear component $\left[F(1,3)=873.44, M S_{\mathrm{e}}=.001\right]$, and intro-

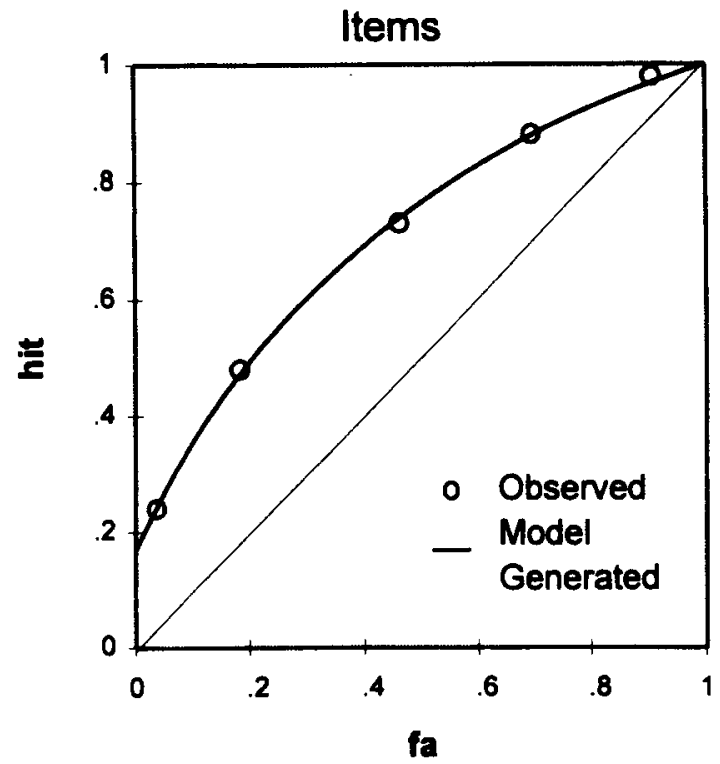

ducing the quadratic component did not lead to a significant improvement in the fit of the equation $[F(2,3)=$ $\left.4.00, M S_{\mathrm{e}}=.001\right]$. Thus, as in the two previous experiments, the item ROC was curvilinear, and the associative ROC was linear.

To determine whether the ROCs could be described with a signal detection model, the functions were plotted in $z$-space (Figure 3). Examination of Figure 3 shows that the item $z$-ROCs exhibited a slight U-shape, and the associative $z$-ROC exhibited a pronounced U-shape. $R^{2}$ values for the $z$-ROCs are presented in Table 2. For the item $z$-ROC, there was a significant linear component $\left[F(1,3)=568.83, M S_{\mathrm{e}}=.001\right]$, and introducing the quadratic did not lead to a significant improvement in the fit of the equation $\left[F(1,2)=10.00, M S_{\mathrm{e}}=.001\right]$, suggesting that the ROC did not deviate significantly from linearity. The slope of the item $z$-ROC was .86 . For the associative recognition $z$-ROC, there was a significant linear component $\left[F(1,3)=53.71, M S_{\mathrm{e}}=.005\right]$; introducing the quadratic component led to a significant improvement in the fit of the equation $\left[F(1,2)=24.50, M S_{\mathrm{e}}=.001\right]$, however, showing that the $z$-ROC was curvilinear. As in Experiment 1, the U-shape of the associative ROC indicates that the overall memory distributions for old and new items were not normally distributed, and the results are thus in conflict with the unequal-variance signal detection model.

As in the previous experiments, the dual-process model was fit to the observed ROCs (see Figure 5). An examination of Figure 5 shows that the model provided an accurate account of the item and associative ROCs. The parameter estimates for the average ROCs are presented in Table 2, and the parameter estimates for individual subjects are presented in Table 3. For the average associative ROC, $R_{\mathrm{n}}$ was .16 , representing the probability that new pairs

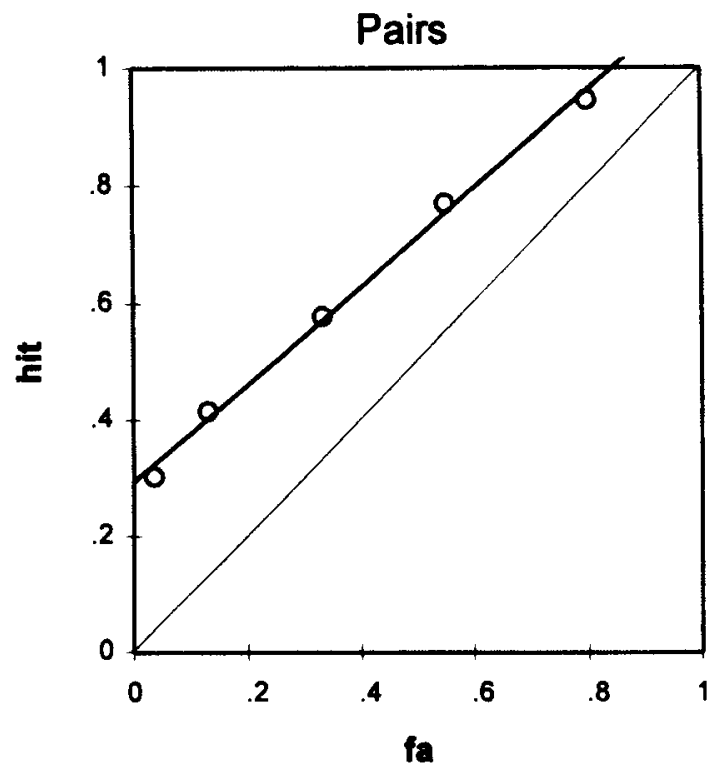

Figure 5. ROCs for item and associative recognition plotted along with the functions generated by the dual-process model for Experiment 3. 
were recollected. As expected, the probability that new pairs were recollected as new was greater than that found in Experiment 1 (.02). Moreover, as in Experiment 2, the single-parameter version of the model $\left(R_{\mathrm{o}}=.34\right)$ could be rejected in favor of the two-parameter model $[F(1,3)=$ $43.55, M S_{\mathrm{e}}=.001$ ], suggesting that $R_{\mathrm{n}}$ did contribute to performance.

Subject ROCs. To examine the effect of averaging across subjects, ROCs were plotted for each subject; they are presented in Figure 6. An examination of the individual ROCs supports the conclusions drawn from the average ROC data; the item ROCs were curvilinear, and the associative ROCs were fit well by linear functions. Linear trend analysis conducted on each subject ROC supported this conclusion. For 5 out of 6 subjects, the associative ROC was linear. Moreover, for 5 out of 6 subjects, the item ROC was curvilinear.

The individual ROCs were also plotted on $z$-coordinates (see Figure 7). An examination of Figure 7 shows that the item $z$-ROCs were linear, and the associative $z$-ROCs were U-shaped. Linearity analysis showed that five out of six item ROCs were linear, and five out of six associative ROCs were curvilinear.

The analysis of individual ROCs led to the same conclusions as did the average ROCs, showing that averaging across subjects was not responsible for the different pattern of results observed for the item and associative ROCs.

In sum, the results of Experiment 3 replicated those of Experiments 1 and 2 in showing that although the item ROCs are curvilinear, associative ROCs are linear. Moreover, when subjects were likely to recollect that new pairs were new, the associative ROC approached the upper $x$-axis at a point less than 1.0. The model analyses showed that the dual-process model provided an accurate account of the item and associative ROCs and that $R_{\mathrm{n}}$ was greater than 0 . It also showed that the average ROCs were representative of the individual subject ROCs. Finally, the U-shaped $z$-ROCs indicated that the signal detection process alone was not sufficient to account for the ROC data.

\section{GENERAL DISCUSSION}

The results of Experiments 1-3 show that ROCs for item judgments are curvilinear, but that ROCs for associative judgments are linear. These results were observed with the average and individual subject ROCs. The curvilinear item ROCs were similar to those found in many previous studies of item recognition and are to be expected if recognition judgments rely on a combination of recollection and familiarity. In contrast, the linear associative ROCs that were observed were predicted on the basis of the assumption that associative recognition judgments rely on recollection. Further support for the notion that associative judgments are based on recollection was gained by varying the probability that subjects could recollect that new pairs were new. Under condi- tions in which it was difficult to recollect rearranged pairs as new (Experiment 1), the ROC approached the 1,1 intercept and $R_{\mathrm{n}}$ was close to zero, suggesting that such recollection occurred very infrequently. However, under conditions in which such recollection was made less difficult (Experiments 2 and 3), the function did not approach the 1,1 intercept and $R_{\mathrm{n}}$ was greater than 0 , suggesting that subjects could recollect that rearranged pairs were not in the study list.

The item recognition ROCs in all three experiments were in general agreement with both the dual-process model and the unequal-variance signal detection model. However, the associative ROCs were linear in probability space and were generally U-shaped in $z$-space. Thus, associative ROCs were as predicted by the dual-process model and were in conflict with the unequal-variance signal detection model. The associative $z$-ROC in Experiment 2 exhibited a slight U-shape, but it was not sufficient for the signal detection model to be rejected. In Experiments 1 and 3, however, the U-shape of the associative $z$-ROCs were sufficient to reject such a model.

Given the results of the present experiments, one could maintain an unequal-variance signal detection model for item recognition and propose some alternative model to account for associative recognition. However, the dualprocess model is preferable because it provides a unified account of both types of recognition memory. Moreover, other studies have shown that even for item recognition, there are conditions in which U-shaped $z$-ROCs are observed (e.g., Yonelinas et al., 1996). Thus, even in tests of item recognition, the dual-process model would seem to be more appropriate than the unequal-variance signal detection model.

It is important to note that these results do not rule out all signal detection models; it may be possible to develop more complex signal detection based models that are in agreement with the data. For example, as a potential extension of the dual-process model, one could assume that both recollection and familiarity reflect a signal detection process (for a discussion of multidimensional signal detection models, see Macmillan \& Creelman, 1991), and such a model might be able to produce the types of ROCs observed. The present results are important in showing that there is a relatively simple dual-process model that can account for item and associative recognition memory, and that the unequal-variance model that is often used has some important limitations.

\section{Did Subjects Recollect New Items as New?}

The results of the present experiments suggest that, under some conditions, subjects recollected that rearranged pairs were not in the study list. However, an informal examination of the item ROCs suggests that subjects did not often recollect that new items were new. Given that the item ROCs were curvilinear, it is not possible to derive estimates for $R_{\mathrm{o}}$ and $R_{\mathrm{n}}$ as was done for the associative ROCs. However, extrapolating the functions to find the intercepts should serve a similar pur- 

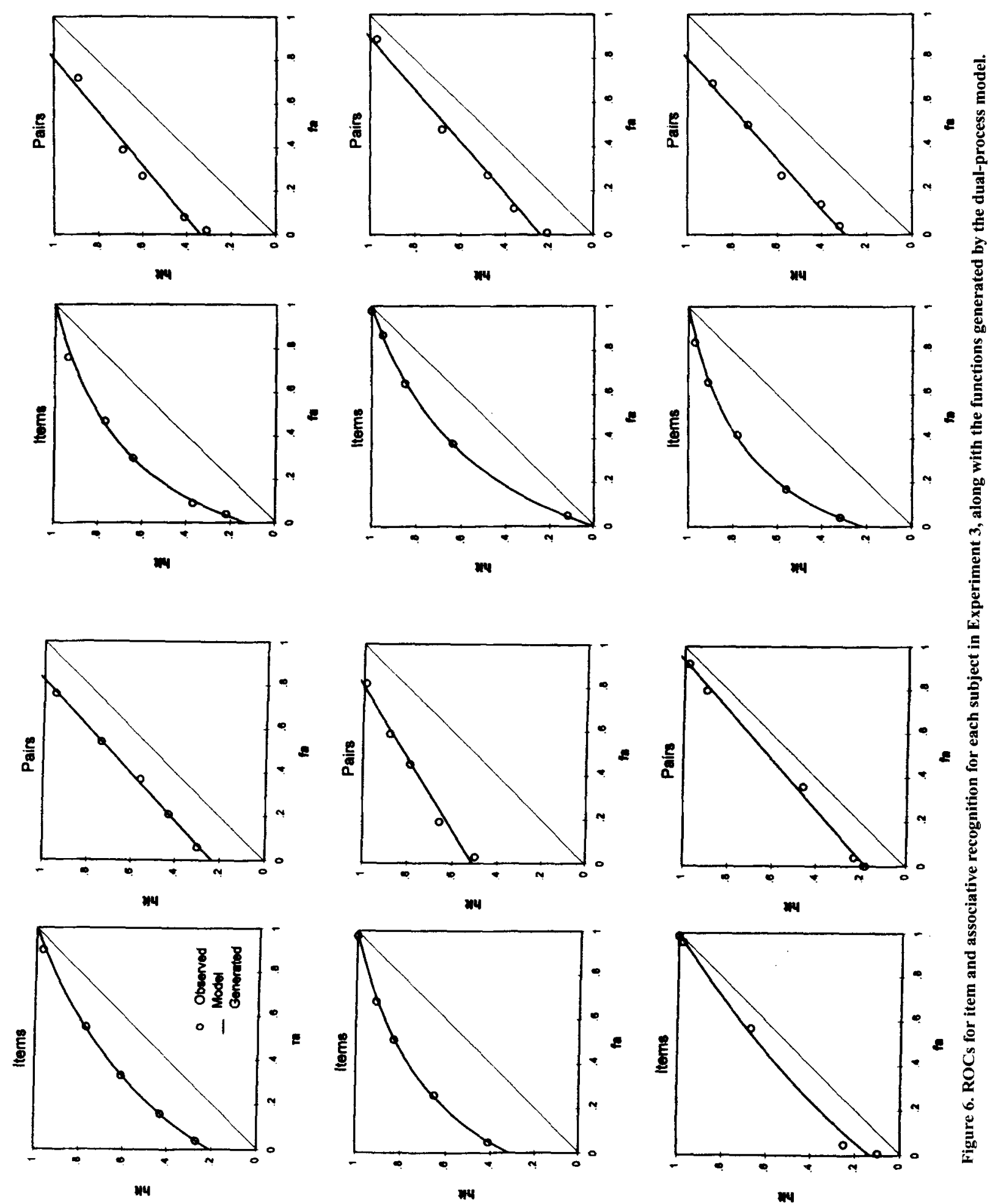
Table 3

Estimated Parameter Values for Item and Associative ROCs for Each Subject in Experiment 3

\begin{tabular}{ccccc}
\hline & \multicolumn{3}{c}{ Condition } \\
\cline { 2 - 3 } Subject & $R_{\mathrm{o}}$ & $d^{\prime}$ & & \multicolumn{2}{c}{ Associative } \\
\cline { 2 - 5 } & .19 & 0.47 & .23 & $R_{\mathrm{o}}$ \\
\hline 1 & .29 & 0.67 & .52 & .18 \\
2 & .12 & 0.22 & .18 & .06 \\
3 & .10 & 0.77 & .34 & .19 \\
4 & .00 & 0.64 & .23 & .11 \\
5 & .19 & 0.82 & .30 & .20 \\
6 &. & &
\end{tabular}

Note-For item recognition, $d^{\prime}$ refers to the distance between the means of the old and new item familiarity distributions, and $R_{\mathrm{o}}$ refers to the probability of recollecting an old item. For associative recognition, $R_{0}$ refers to the probability of recollecting an old pair as old, and $R_{\mathrm{n}}$ refers to the probability of recollecting a new pair as new (i.e., recollecting that the items were not paired together at study).

pose. For the item ROCs in all three experiments, as the hit rate increased, the functions approached 1,1 , which is to be expected if new items are not recollected.

That the dual-process model (Equation 3) provided a good fit for the item ROCs provides further support for the notion that subjects did not often recollect that new items were new. The equation assumes that subjects can recollect old items but that they do not recollect new items. Moreover, subsequent analyses showed that introducing the $R_{\mathrm{n}}$ term to the recognition equation did not lead to a significant improvement in the fit of the model in any of the three experiments, showing that the contribution of $R_{\mathrm{n}}$ to item recognition was negligible. However, it may be possible to find conditions under which subjects are able to recollect new items as new, and under such conditions, it may be necessary to introduce $R_{\mathrm{n}}$ into the model.

\section{Are Associative ROCs Always Linear?}

In contrast to the item ROCs, the associative ROCs in the present experiments were fit well by linear functions. However, there was a noticeable, although nonsignificant, curve to the associative ROC in Experiment 2, and there was one subject in Experiment 3 who exhibited a significantly curvilinear associative ROC. Deviations from linearity may arise for several reasons.

It seems likely that associative judgments may sometimes benefit from familiarity, and thus the observed ROCs should deviate slightly from linearity. First, if related word pairs were used rather than randomly paired words, associative judgments might benefit from assessments of familiarity. For example, it is likely that the word pair grape fruit would be more familiar if it had been studied as a pair than if the words had been presented in different pairs. The reason is that the concept grapefruit might become familiar, in addition to the individual words grape and fruit. If this is true, familiarity might contribute to associative judgments and the ROC would become curved. It is possible that even with the random pairing of words in the present study, some paired words were related, and this might have led to the slight curve that was sometimes observed. Alternatively, there might be conditions under which randomly paired words could be bound in such a way that the pair itself became familiar. Finally, discrepancies in familiarity might sometimes be used to make associative judgments. That is, if one item in a test pair appeared familiar and the other item was much less familiar, a subject might conclude
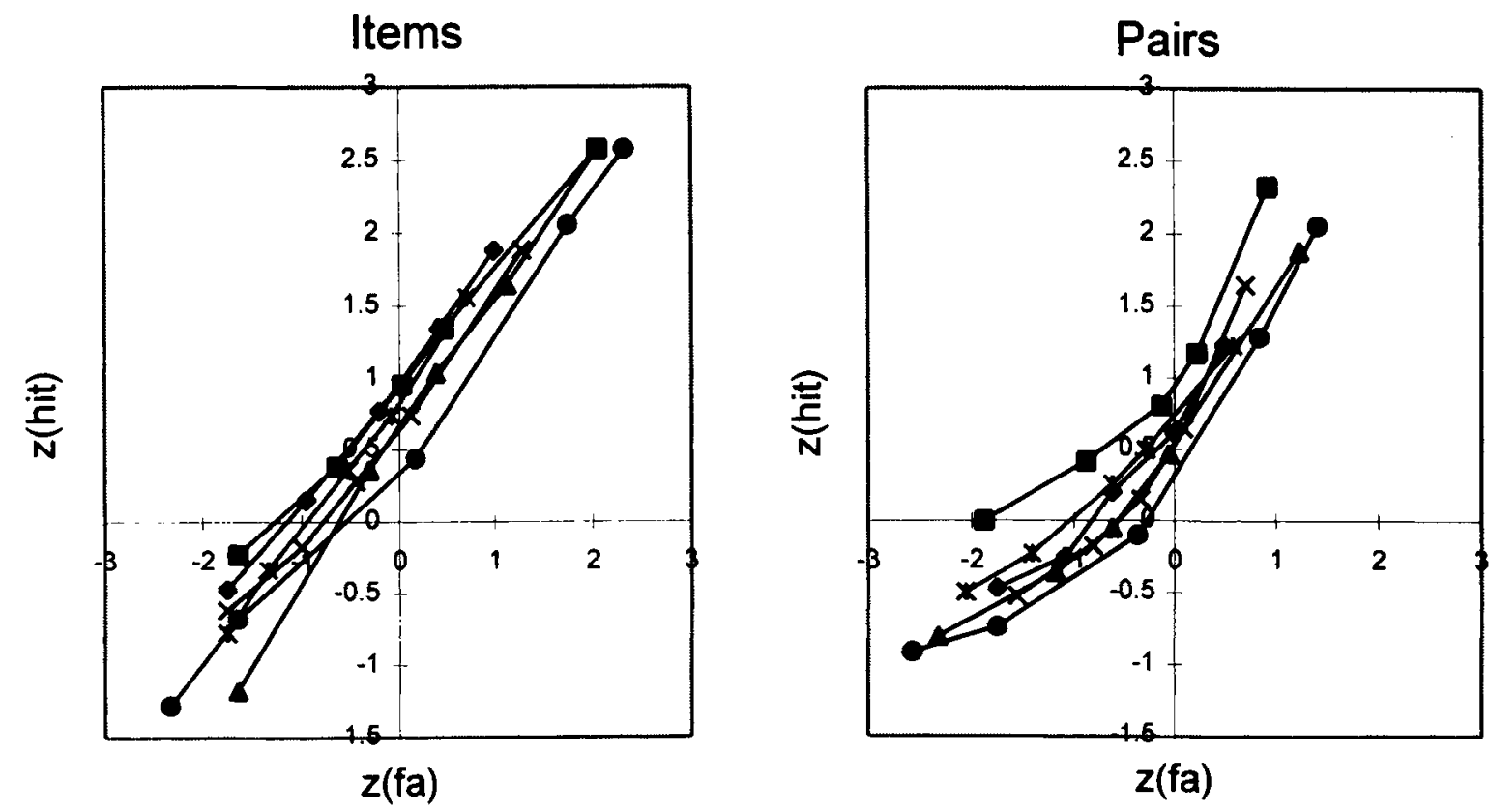

Figure 7. z-ROCs for item and associative recognition for each subject in Experiment 3. 
that the more familiar item was presented very recently and the less familiar item was presented less recently. In this way rearranged pairs might be rejected on the basis of discrepancies in familiarity, and this might also lead to a slight curvilinearity in the associative ROC.

That the observed associative ROCs were fit well by linear functions suggests that those judgments were based primarily on recollection. Further studies are under way, however, to determine whether more curved associative ROCs can be observed under conditions that promote familiarity-based associative judgments.

\section{Global Memory Models}

Hockley (1991), Clark (1992), and Clark and Gronlund (1996) have shown that dissociations between item and associative information pose problems for global memory models. Moreover, none of the global models can account for the pattern of observed slope $\times$ intercept relationships observed for item recognition $z$-ROCs (see Ratcliff et al., 1992; Yonelinas, 1994). At least as problematic, however, is the shape of the associative ROCs observed in the present study. The global memory models generate approximately normal distributions for old and new items and thus predict curvilinear ROCs (see Ratcliff et al., 1994) that will be linear in $z$-space. Because the models assume that the same retrieval and decision processes underlie both item and associative recognition, the ROCs for both types of information should be curvilinear. Examination of the associative ROCs in the present experiments showed, however, that the overall old and new distributions could not have been normal.

The inability of the models to account for the range of ROC results may lie in the assumption, made by all of the models, that recognition judgments are based solely on the assessment of a single familiarity process. One option would be to drop the single-process assumption by introducing a second mechanism qualitatively different from the familiarity mechanism. In fact, all of these models do possess recall-like search mechanisms that could be incorporated into recognition.

One model that may be particularly well suited to the ROC data is TODAM (Murdock, 1982). TODAM assumes that items, which are represented as vectors, are encoded in a distributed manner across a common memory vector. The memory strength (or familiarity) of an item is determined by taking the dot product of the item and memory vector. Studied items tend to lead to higher levels of familiarity than do new items. This model predicts normal familiarity distributions, but unlike the other global memory models, it produces old and new familiarity distributions that are approximately equal in variance. Thus it would be in agreement with the dual-process model with respect to the familiarity component. Moreover, recent simulations done with the TODAM model (Kahana, 1996) show that item familiarity may be independent of recall, which is in general agreement with the assumption that recollection and familiarity are independent. Whether the recall process can be introduced in such a way that allows the model to account for both item and associative recognition ROCs, however, is not yet clear.

\section{CONCLUSION}

The results of the present experiments join a body of literature showing that recognition judgments for item and associative information are dissociated. As previously discussed, item and associative judgments differ in terms of processing time and forgetting rate, and in how they are affected by word frequency and amnesia. The observed ROCs were in agreement with predictions of the dualprocess model and suggest that the differences between item and associative judgments arise because they rely differentially on recollection and familiarity. Because item judgments rely on recollection as well as on familiarity, the asymmetrical curvilinear item ROCs were expected. Because associative judgments rely primarily on recollection, the linear associative ROCs were also expected.

\section{REFERENCES}

ANDERSON, J. R. (1974). Retrieval of prepositional information from long-term memory. Cognitive Psychology, 6, 451-474.

ATKINSON, R. C., \& Juol A, J. F. (1974). Search and decision processes in recognition memory. In D. H. Krantz, R. C. Atkinson, R. D. Luce. \& P. Suppes (Eds.), Contemporary developments in mathematical psychology: Vol.1. Learning, Memory \& Thinking (pp. 243-293). San Francisco: W. H. Freeman.

Banks, W. P. (1970). Signal detection theory and human memory. Psychological Bulletin, 74, 81-99.

ClarK, S. E. (1992). Word frequency effects in associative and item recognition. Memory \& Cognition, 20, 231-243.

Clark, S. E., \& Gronlund, S. D. (1996). Global matching models of recognition memory: How the models match the data. Psychonomic Bulletin \& Review, 3, 37-60.

Clark, S. E., \& Shiffrin, R. M. (1992). Cuing effects and associative information in recognition memory. Memory \& Cognition, 20. 580-598.

Cowan, N., \& StadLer, M. A. (1996). Estimating unconscious processes: Implications of a general class of models. Journal of Experimental Psychology: General, 125, 195-200.

Donaldson, W. (1996). The role of decision processes in remembering and knowing. Memory \& Cognition, 24, 523-533.

Donaldson, W., \& Murdock, B. B. (1968). Criterion change in continuous recognition memory. Journal of Experimental Psychology, 76, 325-330.

Düzel, E., Yonelinas, A. P., Mangun, G. R., Heinze, H., \& TulVING, E. (1997). Event-related brain potential correlates of two states of conscious awareness in memory. Proceedings of the National Academy of Sciences, 94, 5973-5978.

EGAN, J. P. (1958). Recognition memory and the operating characteristic (Tech. Note AFCRC-TN-58-51). Bloomington: Indiana University, Hearing and Communication Laboratory.

ElLIoTT, P. B. (1964). Tables for $d^{\prime}$. In A. S. Swets (Ed.), Signal detection and recognition by human observers: Contemporary readings (pp. 651-684). New York: Wiley.

GaRDINER, J. M. ( 1988). Functional aspects of recollective experience. Memory \& Cognition, 16, 309-313.

GARDINER, J. M., \& JAVA, R. I. (1993). Recognizing and remembering. In A. Collins, M. A. Conway, S. E. Gathercole, \& P. E. Morris (Eds.), Theories of memory (pp. 163-188). Hillsdale, NJ: Erlbaum.

Gillund, G., \& ShifFrin, R. M. (1984). A retrieval model of both recognition and recall. Psychological Review, 91, 1-67.

Glanzer, M., \& Adams, J. K. (1990). The mirror effect in recognition memory: Data and theory. Journal of Experimental Psychology: Learning, Memory. \& Cognition, 16, 5-16. 
Green, D. M., \& Swets. J. A. (1966). Signal detection theory and psychophysics. New York: Wiley.

Gronlund, S. D., \& Elam, L. E. (1994). List-length effect: Recognition accuracy and variance of underlying distributions. Journal of Experimental Psychology: Learning, Memory, \& Cognition, 20, 1355-1369.

Gronlund, S. D., \& RatclifF, R. (1989). Time course of item and associative information: Implications for global memory models. Journal of Experimental Psychology: Learning, Memory, \& Cognition, $15,846-858$.

HintzMan, D. L. (1986). "Schema abstraction" in a multiple-trace memory model. Psychological Review, 93, 411-428.

HOCKLEY, W. E. (1991). Recognition memory for item and associative information: A comparison of forgetting rates. In W. E. Hockley \& S. Lewandowsky (Eds.), Relating theory and data: Essays on human memory in honor of Bennet B. Murdock (pp. 227-248). Hillsdale, NJ: Erlbaum.

HOCKLEY, W. E. (1992). Item versus associative information: Further comparisons of forgetting rates. Journal of Experimental Psychology: Learning, Memory, \& Cognition, 18, 1321-1330.

HOCKLEY, W. E. (1994). Reflections of the mirror effect for item and associative recognition. Memory \& Cognition, 22, 713-722.

HuMPHREYS, M. S. (1978). Item and relational information: A case for context independent retrieval. Journal of Verbal Learning \& Verbal Behavior, 17, 175-187.

HUPPERT, F., \& PIERCY, M. (1976). Recognition memory in amnesic patients: Effects of temporal context and familiarity of material. Cortex, 12, 3-20

JACOBY, L. L. (1991). A process dissociation framework: Separating automatic from intentional uses of memory. Journal of Memory \& Language, 30, 513-541.

JACOBY, L. L., \& DALLAS, M. (1981). On the relationship between autobiographical memory and perceptual learning. Journal of Experimental Psychology: General, 3, 306-340.

JaCOBY, L. L., TotH, J. P., \& Yonelinas, A. P. (1993). Separating conscious and unconscious influences of memory: Measuring recollection. Journal of Experimental Psychology: General, 122, 139-154.

JaCoBy, L. L., TOTH, J. P., Yonelinas, A. P., \& DeBner, J. A. (1994). The relationship between conscious and unconscious influences: Independence or redundancy? Journal of Experimental Psychology. General, 123, 216-219.

JaCoBy, L. L., Yonelinas, A. P., \& Jennings, J. M. (1997). The relationship between conscious and unconscious (automatic) influences: A declaration of independence. In J. Cohen \& J. W. Schooler (Eds.), Scientific approaches to consciousness (pp. 13-47). Mahwah, NJ Erlbaum.

JENNings, J. M., \& JACOBY, L. L. (1993). Automatic versus intentional uses of memory: Aging, attention, and control. Psychology \& Aging, 8, 283-293

JOORDENS, S., \& MERIKLE, P. M. (1993). Independence or redundancy? Two models of conscious and unconscious influences. Journal of $E x$ perimental Psychology: General, 122, 462-467.

KAHANA, M. J. (1996). An analysis of the recognition-recall relationship in four distributed memory models. Manuscript submitted for publication.

Macmillan, N. A., \& Creelman, C. D. (1991). Detection theory: A user's guide. New York: Cambridge University Press.

MANDLER, G. (1980). Recognizing: The judgment of previous occurrence. Psychological Review, 87, 252-271.

MANDLER, G., \& BoECK, W. J. (1974). Retrieval processes in recognition. Memory \& Cognition, 2, 613-615.

Murdock, B. B.. JR. (1965). Signal-detection theory and short-term memory. Journal of Experimental Psychology, 70, 443-447.
Murdock, B. B., JR. (1974). Human memory: Theory and data. New York: Wiley, Halsted Press.

Murdock, B. B. (1982). A theory for the storage and retrieval of item and associative information. Psychological Review, 89, 609-626.

Ratcliff, R., MCKoON, G., \& TINDALl, M. (1994). Empirical generality of data from recognition memory receiver-operating characteristic functions and implications for the global memory models. Journal of Experimental Psychology: Learning, Memory, \& Cognition, 20, 763-785.

Ratcliff, R., Sheu, C.-F., \& Gronlund, S. D. (1992). Testing global memory models using ROC curves. Psychological Review, 99 518-535.

Richardson-KlaVehn, A., \& BjoRK, P. M. (1988). Measures of memory. Annual Review of Psychology, 39, 475-543.

RoEDIGER, H. L., III, \& MCDERMOTT, K. B. (1993). Implicit memory in normal human subjects. In H. Spinnier \& F. Boller (Eds.), Handbook of neuropsychology (Vol. 8, pp. 62-131). Amsterdam: Elsevier.

SCHACTER, D. L., \& GRAF, P. (1986). Preserved learning in amnesic patients: Perspectives from research on direct priming. Journal of Clinical \& Experimental Neuropsychology, 8, 727-743.

SMITH, M. E. (1993). Neurophysiological manifestations of recollective experience during recognition memory judgments. Journal of $\mathrm{Cog}$ nitive Neuroscience, 5, 1-13.

Swets, J. A., TANner, W. P., \& Birdsall, T. G. (1961). Decision processes in perception. Psychological Review, 68, 301-340.

Tотн, J. P. (1996). Conceptual automaticity in recognition memory: Levels-of-processing effects on familiarity. Canadian Journal of Experimental Psychology, 50, 123-138.

Tulving, E. (1985). Memory and consciousness. Canadian Psychologist, 26, 1-12.

Verfaellie, M., \& TREAdWEll, J. R. (1993). The status of recognition memory in amnesia. Neuropsychology, 1, 5-13.

WiCKELGREN, W. A., \& NoRMAN, D. A. (1966). Strength models and serial position in short-term recognition memory. Journal of Mathematical Psychology, 3, 316-347.

WILDING, E. L., \& RUGG, M. D. (1994, November). Event-related potentials dissociate recognition memory with and without retrieval of study context. Paper presented at the 35th Annual Meeting of the Psychonomic Society.

WOLFORD, G. (1971). Function of distinct associations for pairedassociate performance. Psychological Review, 78, 303-313.

YonELINAS, A. P. (1994). Receiver-operating characteristics in recognition memory: Evidence for a dual-process model. Journal of Experimental Psychology: Learning, Memory, \& Cognition, 20, 13411354

Yonelinas, A. P., Dobbins, I., Szymanski, M. D., Dhaliwal, H. S., \& KING, L. (1996). Signal detection, threshold, and dual-process models of recognition memory: ROCs and conscious recollection. Consciousness \& Cognition, 5, 418-441

Yonelinas, A. P., \& JACOBY, L. L. (1994). Dissociations of processes in recognition memory: Effects of interference and of response speed. Canadian Journal of Experimental Psvchology, 48, 516-534.

YONELINAS, A. P., \& JACOBY, L. L. (1995). The relation between remembering and knowing as a basis for recognition: Effects of size congruency. Journal of Memory \& Language, 34, 622-643.

Yonelinas, A. P., \& JACOBY, L. L. (1996). Noncriterial recollection Familiarity as automatic, irrelevant recollection. Consciousness \& Cognition, 5, 131-141

(Manuscript received June 19, 1996; revision accepted for publication November 17,1996 .) 